\title{
Riscos de Interações Medicamentosas Presentes nos Receituários de Pacientes Hipertensos e Diabéticos: Uma Revisão Bibliográfica
}

\author{
Vilmar Luz ; Matheus Santos Marques ${ }^{2}$; Naila Neves de Jesus ${ }^{3}$
}

Resumo: As doenças crônicas não-transmissíveis (DCNT) com o passar do tempo passaram a assumir maior incidência em detrimento das doenças transmissíveis. Dentre as DCNT mais prevalentes destacamse a hipertensão e o diabetes mellitus. Quanto o uso de medicamentos há uma série de questões a serem avaliadas, e a possível ocorrência de interações medicamentosas é uma delas e deve ser levada em questão, pois a interação medicamentosa indesejável prejudica a adesão e o sucesso da terapia. O presente trabalho objetivou analisar a literatura bibliográfica existente com o intuito de investigar as potenciais interações medicamentosas existentes entre os medicamentos utilizados o tratamento do diabetes mellitus concomitante ao tratamento da hipertensão. Foram analisadas e escolhidas 38 literaturas entre livros, artigos científicos, guidelines, diretrizes que foram utilizadas para compor esse artigo de revisão integrativa.

Palavras-chave: diabetes mellitus, hipertensão arterial, interações medicamentosas.

\section{Risks of Drug Interactions Presented in the Prescriptions for Hypertensive and Diabetic Patients: A Bibliographic Review}

\begin{abstract}
Non-communicable chronic diseases (NCDs) over time have become more prevalent at the expense of communicable diseases. Among the most prevalent CNCDs are hypertension and diabetes mellitus. Regarding the use of medications there are a number of issues to be evaluated, and the possible occurrence of drug interactions is one of them and should be taken into account, since undesirable drug interaction impairs adherence and success of therapy. The objective of this study was to analyze the existing bibliographical literature in order to investigate the potential drug interactions between the drugs used to treat diabetes mellitus concomitant with the treatment of hypertension. Thirty-eight literatures were analyzed and selected among books, scientific articles, guidelines, guidelines that were used to compose this article of integrative review.
\end{abstract}

Keywords: diabetes mellitus, hypertension, drugs interactions.

\footnotetext{
${ }^{1}$ Graduando em Farmácia pela Faculdade Independente do Nordeste. vilmarluz@ hotmail.com;

2 Farmacêutico Bioquímico pela UFBA; Especialista em Saúde Pública pela Facinter. Docente da Faculdade Independente do Nordeste.

${ }^{3}$ Farmacêutica generalista pela Fainor. Especialista em Farmacologia Clínica e Prescrição farmacêutica pelo I-BRas. Farmacêutica Hospitalar do Hospital Municipal Esaú Matos, Vitória da Conquista.
} 


\section{Introdução}

Nas últimas décadas, os estudos epidemiológicos demonstram que está ocorrendo uma grande mudança no perfil etiológico de doenças. As doenças transmissíveis foram as principais responsáveis pela morbidade e mortalidade no mundo. Entretanto, as doenças crônicas nãotransmissíveis (DCNT) passaram a assumir maior incidência em detrimento das doenças transmissíveis (MINISTÉRIO DA SAÚDE, 2017; LIMA et al, 2015).

As DCNTs são doenças multifatoriais que podem ser desenvolvidas ao longo do curso de vida do indivíduo e são de longa duração. Constituem a maior causa de morbimortalidade no mundo, além de serem de causarem grande impacto socioeconômico (DUNCAN et al, 2012; MINISTÉRIO DA SAÚDE, 2017). No Brasil, as DCNT são as principais fontes de carga de doença e políticas importante para sua prevenção e controle vem sido implementadas (SCHMIDT et al, 2011).

A hipertensão arterial é uma condição clínica caracterizada pela elevação sustentada dos níveis pressóricos acima ou igual a 140/90 mmHg (SOCIEDADE BRASILEIRA DE CARDIOLOGIA, 2016). O diabetes mellitus é uma síndrome caracterizada pelo aumento de glicose na corrente sanguínea decorrente de um defeito da produção da insulina por parte das células beta, ou por resistência a esse hormônio (SILVA et al., 2015).

A hipertensão frequentemente é associada a distúrbios metabólicos que se agravam na presença de outros fatores de risco como o diabetes mellitus. Alguns estudos apontam que o diabetes e a hipertensão são comumente associadas e que a prevalência de hipertensão é o dobro em comparação a não diabéticos. Além disso, as doenças cardiovasculares são as principais causas de mortalidade em portadores de diabetes (SOCIEDADE BRASILEIRA DE CARDIOLOGIA, 2016; FREITAS; GARCIA, 2012).

Muitos dos problemas relacionados a medicamentos estão relacionados com as interações medicamentosas. As interações medicamentosas referem-se à interferência de um fármaco na ação de outro, ou de um alimento ou nutriente na ação dos medicamentos. Tais interações podem ser benéficas, como também indesejáveis, pois as mesmas podem reduzir ou aumentar o efeito ou resultado contrário ao esperado, aumento dos efeitos adversos, podendo resultar no fracasso na terapia e na progressão da doença (SEHN et al., 2003).

Em qualquer comunidade, a utilização de medicamentos é um processo social que deve ser supervisionado e acompanhados por profissionais de saúde e a Atenção Farmacêutica pode 
contribuir para a diminuir problemas relacionados a medicamentos e a morbimortalidade relacionada à farmacoterapia, incluindo investigar possíveis interações medicamentosas (GUIMARÃES et al, 2012).

Diante disso, o presente trabalho objetiva analisar a literatura bibliográfica existente com o intuito de investigar as potenciais interações medicamentosas existentes entre os medicamentos utilizados o tratamento do diabetes mellitus concomitante ao tratamento da hipertensão.

\section{Metodologia}

A metodologia empregada para a produção desse artigo foi a revisão bibliográfica do tipo integrativa. A revisão integrativa permite integrar, selecionar e apresentar estudos científicos de diversas metodologias, integrando assim seus resultados e montando comparativos. A revisão integrativa permite combinar dados da literatura empírica e com dados científicos provenientes de pesquisas (UNESP, 2015).

Após minuciosa pesquisa bibliográfica foram selecionados através das bases de dados Pubmed, BVSalud e Google acadêmico, utilizando como filtro publicações dos últimos 11 anos. O fluxo de busca pelas literaturas estão dispostos no quadro 1.

Quadro 1. Fluxo de busca por literaturas relevantes sobre o assunto

\begin{tabular}{|c|c|}
\hline Tipo de literatura & Quantidade encontrada \\
\hline Artigos científicos & 29 \\
\hline Livros & 4 \\
\hline Monografias e trabalhos de conclusão de curso & 2 \\
\hline Diretrizes e Guidelines & 4 \\
\hline Total de Artigos & $\mathbf{3 8}$ \\
\hline
\end{tabular}

Fonte: Pesquisa dos autores

Além dos 28 artigos científicos, foram utilizados na produção da revisão 01 guideline da American College of Cardiology, 01 guideline da International Diabetes Foundation, 01 Diretrizes da Sociedade Brasileira de Diabetes, 01 Diretrizes da Associação Brasileira de Cardiologia e 04 livros totalizando 38 publicações utilizadas. Foram utilizados 14 produções 
científicas de referência para hipertensão arterial, 13 literaturas sobre interações medicamentosas entre os tratamentos medicamentosos para hipertensão e diabetes mellitus e 15 produções científicas sobre o diabetes mellitus.

\section{Resultados e Discussão}

\section{Hipertensão arterial}

A hipertensão arterial sistêmica (HAS) é uma condição clínica caracterizada por níveis pressóricos elevados considerando valores de pressão arterial iguais ou maiores que 140/90 mmHg. É uma patologia multifatorial associada a alterações funcionais e/ou estruturais dos órgãos-alvo (coração, rins, encéfalo e vasos sanguíneos) e as alterações metabólicas que levam a eventos cardiovasculares fatais e não fatais (LINS, 2013).

De acordo com a Sociedade Brasileira de Cardiologia (2016) a pressão arterial é classificada de acordo com o quadro 02.

Quadro 2. Classificação da pressão arterial em adultos de acordo com a Sociedade Brasileira de Cardiologia.

\begin{tabular}{|l|l|l|}
\hline Classificação & PAS (mmhg) & PAD $(\mathrm{mmHg})$ \\
\hline Normal & $\leq 120$ & $\leq 80$ \\
\hline Pré-hipertensão & $121-139$ & $81-89$ \\
\hline Hipertensão grau 1 & $140-159$ & $90-99$ \\
\hline Hipertensão grau 2 & $160-179$ & $100-109$ \\
\hline Hipertensão grau 3 & $\geq 180$ & $\geq 110$ \\
\hline
\end{tabular}

Fonte: Sociedade Brasileira de Cardiologia, 2017.

A American College of Cardiology publicou um guideline em 2017 na qual traz novos parâmetros de valores de pressão arterial, o que muda também a classificação, como demonstrado no quadro 3.

Quadro 3. Classificação da pressão arterial em adultos de acordo com a American College of Cardiology.

\begin{tabular}{|l|l|l|}
\hline Classificação & PAS (mmhg) & PAD $(\mathrm{mmHg})$ \\
\hline Normal & $<120$ & $<80$ \\
\hline Elevada & $120-129$ & $<80$ \\
\hline Hipertensão grau 1 & $130-139$ & $80-89$ \\
\hline Hipertensão grau 2 & $\geq 140$ & $\geq 90$ \\
\hline
\end{tabular}

Fonte: American College of Cardiology, 2017. 
Os valores apresentados pela America College of Cardiology em consonância com a American Heart Associaton, apresenta como pressão arterial elevada ou hipertensão arterial grau 1, valores pressóricos de $130 \mathrm{mmHg}$ ou mais para medida de pressão sistólica ou resultados de $80 \mathrm{mmHg}$ ou mais para pressão diastólica e essa mudança reflete nas complicações cardiovasculares que podem ocorrer em valores pressóricos mais baixos do que 140/90 mmHg (AMERICAN COLLEGE OF CARDIOLOGY, 2017).

A hipertensão é uma das doenças crônicas não transmissíveis (DCNT) mais prevalente no mundo, sendo uma das causas de maior óbito, elevado número de mortes prematuras e perda de qualidade de vida (OLIVEIRA et al, 2017). A prevalência mundial da hipertensão em 200 era de 25\%, sendo sua estimativa para 2025 de 29\% (MACEDO et al, 2017). Fatores como obesidade, tabagismo, consumo excessivo de álcool, inatividade física e alto consumo de sal e gorduras pode aumentar o risco de surgimento da hipertensão arterial (FERREIRA et al., 2017).

De acordo com dados do VIGITEL (2016) o número de pessoas que foram diagnosticadas com hipertensão no Brasil, cresceu 14,2\% entre os anso de 2006 a 2016, sendo que sua prevalência aumenta com a idade avançada e menor escolaridade. Em 2013, a Pesquisa Nacional de Saúde divulgou que 21,4\% dos brasileiros tinham diagnóstico de hipertensão arterial dado pelo médico que os acompanhavam, enquanto 3,0\% da população do país nunca tenha medido a pressão arterial adequadamente.

Apesar de ser tratável, a hipertensão é um caminho que pode levar a doença cardiovascular, ao passo que em 2008, no Brasil, em uma pesquisa feita com 400.000 pessoas, $24,0 \%$ das mulheres e $17,3 \%$ dos homens com idade maior ou igual a 20 anos e cerca de metade dos homens e mulheres com idade igual ou acima de 60 anos relataram diagnóstico prévio de hipertensão (SCHMIDT et al., 2011).

A hipertensão é uma doença silenciosa apresentando sintomas que podem ser confundidos com outras doenças e sintomas autolimitados por serem inespecíficos, como: cefaleia pulsátil que ocorre nas primeiras horas da manhã e desaparece ao decorrer do dia, sede e dor na nuca (OIGMAN, 2014).

A hipertensão é considerada um grave problema de saúde pública, sendo responsável por uma porcentagem alta de internações e aumento dos custos médicos e assistenciais para o governo, além de ser um importante fator de risco para doenças cardiovasculares e complicações como acidente vascular cerebral, infarto agudo do miocárdio, doenças renais 
crônicas, insuficiência cardíaca, doença arterial coronariana, entre outras (FERREIRA et al., 2017).

\section{Tratamento medicamentoso}

O objetivo do tratamento farmacológico para a hipertensão é reduzir os níveis de pressão arterial associado a mortalidade e morbidade. Após a falha do tratamento não medicamentoso, a primeira escolha de tratamento é com agentes da classe dos diuréticos, inibidores da enzima conversora de angiotensina, bloqueadores de canais de cálcio (DIPIRO et al.,2008)

Os diuréticos são os anti-hipertensivos mais utilizados devido ao seu baixo custo e eficácia terapêutica. Agem geralmente aumentando a excreção do volume urinário, principalmente, sódio e água, diminuindo a resistência vascular periférica e consequentemente, diminui os níveis pressóricos (MARTELLI, LONGO, SERIANI, 2008).

Estão inseridos na classe dos fármacos diuréticos, os tiazídicos, sendo o mais utilizado dessa classe, a hidroclorotiazida; os diuréticos poupadores de potássio como uma alternativa viável para impedir a secreção de íons potássio através do bloqueio da ação da aldosterona, como por exemplo, a espironolactona, ou por inibir o influxo de sódio através dos canais iônicos na membrana luminal (exemplo: amilorida e triantereno) e os diuréticos da alça como a furosemida, responsáveis por inibir a reabsorção de sódio (KATZUNG; MASTERS; TREVOR, 2017; KHANGURA et al., 2018).

Os medicamentos pertencentes a classe dos betabloqueadores é responsável por antagonizar os efeitos das catecolaminas em adrenoreceptores beta. Pertencem a essa classe o propranolol, labetalol, atenolol, nebivolol,metoprolol e o timolol (KATZUNG MASTERS; TREVOR,2017). Os beta-bloqueadores apresentam propriedade antiarrítmicas e antianginosas, úteis para pacientes hipertensos que possuem comorbidades (MARTELLI, LONGO, SERIANI, 2008).

Os bloqueadores dos canais de cálcio agem bloqueando os canais de cálcio, reduzindo a resistência vascular periférica através da depleção de concentração de cálcio nas células de músculo liso. Fazem parte dessa classe o verapamil, diltiazem, anlodipino e o nifedipino (BARROS, 2016). 


\section{Diabetes mellitus}

O diabetes mellitus (DM) é uma doença caracterizada por hiperglicemia e alterações no metabolismo dos macronutrientes (lipídeos, carboidratos e proteínas), decorrente de uma depleção de insulina ou de uma incapacidade desse hormônio de exercer suas funções no organismo. Apresenta como sintomas, a polidpsia, poliúria, borramento da visão e perda de peso (DIAS et al., 2016).

O diabetes mellitus é uma doença crônica não transmissível de alta prevalência mundial associada a fatores como: crescente urbanização, transições epidemiológica, demográfica e nutricional, sedentarismo, excesso de peso e maior sobrevida de portadores de diabetes (SOCIEDADE BRASILEIRA DE DIABETES, 2017).

Segundo a Federação Internacional de Diabetes - IDF (2017), 425 milhões de pessoas vivem com diabetes e estima-se que esse numa aumente $48 \%$ até 2045. No Brasil, em 2013, Pesquisa Nacional em Saúde, a prevalência de brasileiros diagnosticados com diabetes era de $6,2 \%, 5,4 \%$ no gênero masculino e 7,0\% no gênero feminino. A doença cardiovascular é a principal causa de morte entre os pacientes diabéticos, possivelmente associada as complicações advindas da doença (doenças micro e macrovasculares, nefropatia, neuropatia, pé diabético, retinopatia, doença cerebrovascular, cardiovascular ou arterial periférica) (SOCIEDADE BRASILEIRA DE DIABETES, 2017).

O diabetes mellitus se apresenta em dois subtipos principais: tipo I e tipo II. O diabetes mellitus tipo I é caracterizado pela destruição das células secretoras de insulina, através de mecanismos autoimunes, o que causa depleção de secreção do hormônio insulina, enquanto o diabetes mellitus tipo II é caracterizado por uma resistência dos tecidos-alvo ao hormônio insulina; ambos mecanismos promovem o mesmo efeito, a hiperglicemia (OLIVEIRA et al., 2015; COSTA et al., 2016).

A classificação do estado diabético se dá através de parâmetros laboratoriais como glicemia em jejum, hemoglobina glicada (Hb1Ac), glicemia pós-prandial e teste de tolerância à glicose (TOTG) (SOCIEDADE BRASILEIRA DE DIABETES, 2017). No quadro 04 tem a classificação do diagnóstico laboratorial pela glicemia em jejum. 
Quadro 04. Classificação do diabetes mellitus de acordo diagnóstico laboratorial.

\begin{tabular}{|c|c|}
\hline DIAGNÓSTICO & GLICEMIA EM JEJUM (mg/dL) \\
\hline Normoglicemia & $<100$ \\
\hline $\begin{array}{c}\text { Pré-diabetes ou risco aumentado para } \\
\text { DM (glicemia de jejum alterada) }\end{array}$ & $\geq 100 \mathrm{e}<126$ \\
\hline Diabetes estabelecido & $\geq 126$ \\
\hline
\end{tabular}

Fonte: Sociedade Brasileira de Diabetes, 2017.

\section{Tratamento medicamentoso}

O tratamento do paciente diabético visa atingir objetivos definidos como glicemia de jejum menor que $126 \mathrm{mg} / \mathrm{dL}$ e percentual de Hb1Ac menor que 7\% (MARRERO,2015).

O tratamento medicamentoso é realizado através das seguintes classes de medicamentos: biguanidas, tiazolidinedionas, sulfoniluréias, glinidas, inibidores da alfa glicosidase, análogo do peptídeo 1 semelhante ao glucagon (aGLP-1) e inibidores a dipeptidil peptididase (iDPP-4), amilinomiméticos, Inibidores do cotransporte sódio-glicose (SGLT2) e a insulina (SILVEIRO; SATLER, 2015). O mecanismo de ação e os representantes de cada classe estão dispostos na tabela 01 .

Tabela 01. Categorias de hipoglicemiantes com mecanismos de ação e representantes de cada classe.

\begin{tabular}{c|c|c}
\hline Classe medicamentosa & Mecanismo de ação & Fármacos \\
\hline Biguanidas & $\begin{array}{c}\text { Aumento da ação da insulina } \\
\text { por diminuir a produção } \\
\text { hepática de glicose. }\end{array}$ & Metformina \\
\hline Sulfoniluréias & $\begin{array}{c}\text { Aumento da secreção de } \\
\text { insulina }\end{array}$ & $\begin{array}{c}\text { Glibenclamida, } \\
\text { Glimepirida, Gliclazida, } \\
\text { Glipizida }\end{array}$ \\
\hline Tiazolidinediona & $\begin{array}{c}\text { Aumento da sensibilidade à } \\
\text { insulina em tecidos periféricos }\end{array}$ & Pioglitazona \\
\hline $\begin{array}{c}\text { Inibidores da alfa- } \\
\text { glicosidase }\end{array}$ & $\begin{array}{c}\text { Retardo na absorção de } \\
\text { carboidratos }\end{array}$ & Acarbose \\
\hline Análogos GLP-1 & $\begin{array}{c}\text { Retardo da absorção da glicose } \\
\text { e esvaziamento gástrico; } \\
\text { aumento da secreção de } \\
\text { insulina e diminuição de } \\
\text { secreção do glucagon }\end{array}$ & Exetanida, Liraglutida \\
\hline iDPP-4 & $\begin{array}{c}\text { Incretinomiméticos; aumenta } \\
\text { secreção de glicose e diminui } \\
\text { de glucagon. }\end{array}$ & $\begin{array}{c}\text { Sitagliptina, } \\
\text { Vildagliptina, }\end{array}$ \\
& Saxagliptina, Linagliptina. \\
\hline
\end{tabular}




\begin{tabular}{c|c|c}
\hline Amilinomiméticos & $\begin{array}{c}\text { Redução da secreção de } \\
\text { glucagon e promove a } \\
\text { lentificação o esvaziamento } \\
\text { gástrico }\end{array}$ & Pramlintida \\
\hline $\begin{array}{c}\text { Inibidores do cotransporte } \\
\text { sódio-glicose }\end{array}$ & $\begin{array}{c}\text { Inibe a reabsorção de glicose } \\
\text { nos rins }\end{array}$ & $\begin{array}{c}\text { Dapaglifozina, } \\
\text { Empaglifozina, } \\
\text { Canaglifozina }\end{array}$ \\
\hline Insulinas & $\begin{array}{c}\text { Suplemento a produção } \\
\text { endógena de insulina }\end{array}$ & $\begin{array}{c}\text { NPH,Regular, Detemir, } \\
\text { Aspart, Lispro, Glargina }\end{array}$ \\
\hline
\end{tabular}

Fonte: SILVEIRO; SATLER, 2015.

O tratamento farmacológico para o portador de diabetes mellitus tipo II muitas vezes não é apenas para o controle da glicemia devido ao fato do diabetes vir acompanhado de comorbidades como: hipertensão arterial sistêmica, obesidade e dislipidemia (GUIDONI et al., 2009).

A obesidade e uma patologia bastante prevalente em pacientes portadores de diabetes tipo II. Para o controle do peso, além da dieta hipocalórica e da prática de exercícios físicos, em alguns casos utiliza-se fármacos anti-obesidade como o orlistate e sibutramina, além da fluoxetina e sertralina (GUIDONI et al., 2009).

A hipertensão arterial sistêmica, é usualmente encontrada em individuos que possuem diabetes mellitus tipo II, sendo que sua presença aumenta duas vezes mais os riscos de doenças cardiovasculares e acelera lesões micro e macro vasculares. A estratégia de tratamento engloba medicamentos anti-hipertensivos que agem na inibição do sistema renina- angiotensina, como os inibidores da enzima conversora de angiotensina e antagonistas dos receptores de angiotensina II (GUIDONI et al., 2009).

\section{Problemas relacionados a medicamentos: interações medicamentosas}

Dentre os problemas relacionados a medicamentos, as interações medicamentosas são as mais comuns, principalmente em indivíduos polimedicados. As interações medicamentosas são definidas como a interferência de um fármaco na ação de outro ou de um alimento ou nutriente na ação de medicamentos. Algumas interações visam tratar doenças concomitantes, reduzir efeitos adversos e prolongar a duração do efeito, entre outros. Entretanto, há interações indesejáveis e essas determinam a diminuição do efeito ou resultado inesperado, aumento de 
efeitos adversos e redução na atividade do medicamento e perda da eficácia (SEHN et al., 2003).

Em um estudo feito por Melgaço et al (2011), analisando a polifarmácia de pacientes em uma clínica de cuidados paliativos, observou que das 258 prescrições médicas analisadas há uma média de 10,9 medicamentos por pacientes, comprovando a existência de polifarmácia, que é um dos fatores que potencializam a ocorrência de interações medicamentosas. Estimase que o risco de interações medicamentosas eleva-se praticamente $100 \%$ a partir de 8 medicamentos por prescrição.

\section{Interações medicamentosas potenciais entre anti-hipertensivos e hipoglicemiantes}

Jesus et al (2014) em seu estudo para avaliar as interações medicamentosas envolvendo agentes hipoglicemiantes durante a hospitalização de idosos observou que a metformina apresentou potencial interação com oito fármacos dentre eles, medicamentos pertencentes a classe dos beta-bloqueadores (atenolol, carverdilol e propranolol), provocando aumento na concentração plasmática e absorção de metformina, e o aparecimento de quadros de hipoglicemia e hiperglicemia.

Já a glibenclamida apresentou interação considerável com 12 fármacos sendo o captopril o mais frequente, seguido pelo atenolol, carvedilol, amiodarona, propranolol. didroclorotiazida e enalapril, promovendo a diminuição da eficácia, hipoglicemia excessiva e aumento nos níveis plasmáticos de glibenclamida, (JESUS et al., 2014).

Diversos estudos demonstram que o uso associado de metformina/ glibenclamida/insulina e enalapril/captopril podem elevar o risco de desenvolvimento de hipoglicemia, pois há a suspeita de que ocorra o aumento temporário da sensibilidade à insulina, devido ao captopril (REMPEL et al., 2015, MORENO et al., 2007). No estudo de Amaral e Perassolo (2012) avaliando possíveis interações medicamentosas entre antihipertensivos e antidiabéticos em participantes do grupo HIPERDIA, analisou que o uso de glibenclamida ou metformina associado a hidroclorotiazida pode aumentar a glicemia por diminuir o efeito desses hipoglicemiantes.

Em um estudo feito por Masahiko (2018) analisando o risco de hipoglicemia através do Japanese Adverse Drug Event Report (JADER), observou que o uso concomitante de 
hipoglicemiantes da classe dos inibidores da DPP-4 e anti-hipertensivo da categoria dos bloqueadores de cálcio, provoca hipoglicemia, sendo esse mecanismo ainda desconhecido. Wang et al (2016), observou que o uso de nifedipino concomitante ao uso em monoterapia de uma sulfoniluréia atenua a indução de hiperglicemia ao realizar experimento injetando tais drogas em células da veia umbilical humana.

A metformina ao interagir com o atenolol, anti-hipertensivo pertencente à classe dos betabloqueadores, pode ter seus níveis aumentados por redução de sua eliminação induzido por atenolol, enquanto com metoprolol, outro tipo de betabloqueador pode diminuir os níveis plasmáticos de metformina por induzir a sua metabolização (MAIDEEN; JUMALE; BALASUBRAMANIAM, 2017). Em um estudo analisando 200 prescrições médicas foi observado interação de hidroclorotiazida e metformina em uma mesma prescrição, e essa interação promove hiperglicemia e intolerância a glicose induzida pela hidroclorotiazida (FAROOQUI; KARIM; MUNEER, 2018).

Kothari e Ganguly (2014), em seu estudo observacional concluíram que o atenolol, anlodipino, hidroclorotiazida, furosemida, enalapril, losartana e metformina eram as drogas mais frequentemente associadas a interações medicamento-medicamento. Essas possíveis interações costumam ser frequentes principalmente por que pacientes que apresentam essas comorbidaes (diabetes mellitus e hipertensão), utilizam mais de dois medicamentos, o que comumente denomina-se polifarmácia (SILVA et al., 2015).

\section{Considerações Finais}

Diante do exposto, pode-se averiguar através da pesquisa que portadores de comorbidades como o diabetes mellitus e a hipertensão arterial, cuja prevalência é alta em todo o mundo, aumentam o risco e a chance de ocorrência de interações medicamentosas, que é um sério problema relacionados a medicamentos.

As interações medicamentosas entre os medicamentos utilizados nos tratamentos para hipertensão e diabetes normalmente ocorrem por mecanismos farmacocinéticos e/ou farmacodinâmicos que alteram a excreção, absorção metabolismo, excreção, refletindo assim no aumento ou diminuição dos efeitos clínicos e adversos. 
Uma ferramenta importante para rastrear, investigar e reduzir interações medicamentosas é a atenção Farmacêutica, realizada pelo profissional farmacêutico, na qual o mesmo investiga as possíveis interações, identifica os problemas relacionados a farmacoterapia, e junto com o paciente resolve-os, garantindo assim uma adesão terapêutica e melhores resultados nos objetivos terapêuticos.

\section{Referências}

AMERICAN COLLEGE OF CARDIOLOGY. 2017 Guideline for the prevention, detection, evaluation and management of high blood pressure in adults. N1 ed. 28 p. 2017.

BARROS, T.S. Análise das interações medicamentosas entre anti-hipertensivos, hipoglicemiantes e anti-hiperglicemiantes em diabéticos no Hospital Universitário de Brasília. 56 fl. Monografia Curso de Farmácia, UNB, 2016.

COSTA, J.R.G et al. Educação em saúde sobre atenção alimentar: uma estratégia de intervenção em enfermagem aos portadores de diabetes mellitus. Mostra Interdisciplinar do curso de Enfermagem. v.2, n.1, 2016.

DIAS, O.V et al. Diabetes mellitus em Montes Claros: inquérito de prevalência autorreferida. Revista Brasileira em Promoção da Saúde. v.29, n.3, p.406-413, 2016;

DIPIRO, J.T. et al. Pharmacotherapy: a pathophysiologic approach. USA: The McGraw-Hill. 7.ed. 2008. $2597 \mathrm{p}$.

DUNCAN, B.B et al. Doenças crônicas não transmissíveis no Brasil; prioridade para enfrentamento e investigação. Rev Saúde Pública, v 26, p 126-134, 2012.

FAROOQUI, R. et al. Potential drug-drug interactions among patient's prescriptions collected from Medicine out-patient setting. Pak j Med Sci. v.34, n.1, p.144-148, 2018.

FERREIRA, R.C. et al. Perfil clínico-epidemiológico dos portadores de hipertensão atendidos na atenção básica do estado de Alagoas. Medicina (Ribeirão Preto, Online). v.50, n.6, p.349-357, 2017.

FREITAS, L.R.S; GARCIA, L.P. Evolução da prevalência do diabetes associado à hipertensão arterial no Brasil: análise da Pesquisa Nacional por Amostra de Domicílios, 1998, 2003, 2008. Epidemiol. Serv. Saúde, v. 21, n. 1, p. 7-19, 2012.

GUIDONI, C. M. et al. Assistência ao Diabetes no Sistema Único de Saúde: Análise do Modelo Atual. Brazilian Journal of Pharmaceutical Sciences. v.45 n.1 p. 37-48, 2009

GUIMARÃES, V.G et al. Perfil Farmacoterapêutico de um grupo de idosos assistidos por um programa de Atenção Farmacêutica na Farmácia Popular do Brasil no município de Aracaju SE. Rev Ciênc Farm Básica Apl., v. 33, n.2, 307-312 p, 2012.

INTERNATIONAL DIABETES FOUNDATION. IDF Diabetes Atlas. 8 ed. 2017. 150p.

804 Id on Line Rev. Mult. Psic. V.12, N. 40. 2018 - ISSN 1981-1179 Edição eletrônica em http://idonline.emnuvens.com.br/id 
JESUS, A.P.A. et al. Possíveis interações medicamentosas envolvendo o usp de agentes hipoglicemiantes durante a hospitalização de idosos no sistema único de saúde da região do médio Araguaia. Revista Panorâmica On-line. v.16, p.01-18, 2014;

KATZUNG, B.G; MASTERS, S.B; TREVOR, A.J. Farmacologia Básica e Clínica. Porto Alegre: ARTMED.12 ed. 2017. 1244p.

KHANGURA, D.S. et al. Hypertension in Diabetes. MDText.com. p.8, 2018.

KOTHARI, N; GANGULY, B. Potentional drug-drug interactions among medications prescribed to hypertensive patients. Journal of Clinical and diagnostic research. v. 8, n.11, p. 1-4, 2014.

LIMA, R. F. et al. Interações medicamentosas potenciais em diabéticos tipo 22 participantes de um programa de educação em saúde. Infarma, v. 27, n. 3, p. 160-167, 2015.

LINS, G.A.P. Interações medicamentosas no tratamento de pacientes hipertensos em uma unidade de saúde. 33 fl. Monografia - Departamento de Farmácia, UEPB, 2013.

MACEDO, J.L. et al. Epidemiological profile of arterial hypertension in a maranhense municipal. ReonFacema. v.3, n.4, p.693-698, 2017.

MAIDEEN, N.M.P; JUMALE, A; BALASUBRAMANIAM, R. Drug interactions of metformin involving drug transporter proteins. Adv Pharm Bull. v.7, n.4, p.501-505, 2017.

MARRERO, M.N. Aderência do tratamento em pacientes diabéticos: uma proposta de ação. 15fl. Trabalho de Conclusão de Curso. Especialização em Saúde da Família, UERJ, 2015.

MARTELLI, A; LONGO, M.A; SERIANI, C; Aspectos clínicos e mecanismo de ação das principais classes farmacológicas usadas no tratamento da hipertensão arterial sistêmica. Estud Biol. v.30, n.70, p.149-156, 2008.

MASAHIKO, G. Risk of hypoglycemia after concomitant use of antidiabetic, antihypertensive, and antihyperlipidemic medications: a database study. The Journal of Clinical Pharmacology. v.0, n.0, p. 1-8, 2018.

MELGAÇO, T. B. et al. Polifarmácias e ocorrências de possíveis interações medicamentosas. Rev. Para. Med., v. 25, n. 1, 2011.

MINISTÉRIO DA SAÚDE. Curso: Ações de vigilância e prevenção de doenças crônicas não transmissíveis. Acesso em:10/10/2017 Disponível em: < https://moodle2.ufma.unasus.gov.br/\#/index>

MINISTÉRIO DA SAÚDE. VIGITEL BRASIL 2016. Disponível em < http://portalarquivos.saude.gov.br/images/pdf/2017/abril/17/Vigitel.pdf> Acesso em: 12/05/2018.

MINISTÉRIO DA SAÚDE. Pesquisa Nacional de saúde. 2013 Disponível em < https://www.pns.icict.fiocruz.br/index.php?pag=resultados> Acesso em: 12/05/2018.

MORENO, A.H et al. Atenção farmacêutica na prevenção de interações medicamentosas em hipertensos. Ver Inst Ciênc Saúde, v.25, n.4, p.373-377, 2007.

OIGMAN, W. Sinais e sintomas em hipertensão arterial. JBM, v.102, n.5, p. 13-18, 2014. 
OLIVEIRA, V.S. et al. Análise dos fatores de risco para doenças crônicas não transmissíveis: estudo com colaboradores de uma instituição privada. Santa Maria. v.43, n.1, p. 214-224, 2017.

REMPEL, $C$ et al. Análise da medicação utilizada por diabéticos e hipertensos. Caderno pedagógico, v. 12, n. 1, p. 241-252, 2015.

SEHN, R et al. Interações medicamentosas potenciais em prescrições de pacientes hospitalizados. Infarma, v.15, n. 9-10, p. 77-81, 2003.

SILVA, K.O et al. Avaliação da compreensão dos pacientes portadores do diabetes mellitus tipo II quanto ao tratamento farmacológico e não farmacológico. Rev. Saúde. Com, v.11, n.4, p.382-396, 2015.

SILVEIRO, S.P; SATLER, F. Rotinas em endocrinologia. Porto Alegre: Artmed. 1 ed. 2015. 464 p.

SOCIEDADE BRASILEIRA DE CARDIOLOGIA. $7^{\mathbf{a}}$ Diretriz Brasileira de Hipertensão Arterial. Rio de Janeiro: Arquivos Brasileiros de Cardiologia. v. 107, n. 3, 103 p., 2016.

SOCIEDADE BRASILEIRA DE DIABETES. Diretrizes da Sociedade Brasileira de Diabetes 20172018. São Paulo: Editora Clannad. 2017. 383p.

SCHIMIDT, M.I et al. Doenças crônicas não transmissíveis no Brasil: carga e desafios atuais. The Lancet: Série Saude no Brasil, 14 p., 2011.

UNESP. Tipos de revisões de literatura. 2015. Disponível em: < http://www.fca.unesp.br/Home/Biblioteca/tipos-de-evisao-de-literatura.pdf> Acesso em: 16/05/2018

WANG, L.P. et al. Combination Therapy of Nifedipine and Sulphonylureas Exhibits a Mutual Antagonistic Effect on the Endothelial Cell Dysfunction Induced by Hyperglycemia Linked to Vascular Disease, 2016. Cell Physiol Biochem. 2016; 38(6):2337-47.

\section{Como citar este artigo (Formato ABNT):}

LUZ, Vilmar; MARQUES, Matheus Santos; JESUS, Naila Neves de. Riscos de Interações Medicamentosas Presentes nos Receituários de Pacientes Hipertensos e Diabéticos: Uma Revisão Bibliográfica. Id on Line Rev.Mult. Psic., 2018, vol.12, n.40, p.793-806. ISSN: 1981-1179.

Recebido: $21 / 05 / 2018$

Aceito: 22/05/2018 